\title{
Potential and challenges of testis tissue xenografting from diverse ruminant species
}

\author{
Ali Honaramooz \\ Department of Veterinary Biomedical Sciences, Western College of Veterinary Medicine, University of \\ Saskatchewan, 52 Campus Drive, Saskatoon, Saskatchewan S7N 5B4, Canada
}

\begin{abstract}
Summary
In 2002, we reported that small fragments of testis tissue from immature mouse, pig or goat donors grafted in recipient mice undergo development, maturation and complete spermatogenesis, including the generation of fertilisation-competent murine, porcine or caprine sperm. Testis tissue xenografting (TTX) was then successfully applied using a range of donor species including laboratory/domestic/non-domestic animals and primates. This system offers a novel in vivo model for the study of testis function, and a previously unavailable opportunity to produce sperm in the grafts from immature donors of diverse species. The TTX model also provides easier access for experimental manipulation of the grafted testis tissue or its environment in the recipient mouse; something that is not feasible in many donor species. This application will allow analysis of, for instance, the effects of new hormone regimens, drugs or toxicants on testis function, without experimentation in the target species. Grafting of fresh or preserved testis tissue also can be used as an invaluable tool for the conservation of fertility from immature individuals of valuable or endangered animals. Reviewed here are an overview of the contributions by the author and colleagues and a critical examination of the salient literature on TTX especially using ruminant donors, as well as examples of its variety of current and potential applications for research in male reproductive biology and technologies using ruminant models. The challenges facing optimisation of TTX model as well as its field/experimental uses, along with insights and suggested remedies, are also discussed.
\end{abstract}

\section{Introduction}

Our knowledge of the testicular development and spermatogenesis in humans and large animals, including ruminant species, is relatively limited due to difficulty of conducting in situ studies. Both in vivo and in vitro approaches for the study and manipulation of testis function have, 
therefore, largely relied on laboratory rodent models, the results of which may not necessarily represent the situation in other species.

At various times in the past two centuries, classical experimentations as well as anecdotal reports of transplantation of testes have piqued the interest of scientists, and occasionally the public. Some of these early reports included claims of the presumed rejuvenating effects of testis extracts for aged men, which at best were uncontrolled trials and faded after the 1930s, when they were scientifically discredited and the risk of disease transmission was highlighted. Most reports, however, described well-documented examinations of testis tissue grafting as a tool to study steroidogenesis and spermatogenesis (e.g., Deanesly 1954, Kuopio et al. 1989, Johnson et al. 1996). In fact, Arnold Berthold, perhaps unknowingly, initiated the field of modern endocrinology when in 1849 he showed that replacing the testes back into the abdominal cavity of castrated roosters could lead to restoration of normal male behavior and characteristics (Setchell 1990).

Prior to the $21^{\text {st }}$ century, transplantations of testis tissue were largely limited to reports of autografting and, to a lesser degree, allografting experiments (donor and recipient being genetically non-identical members of the same species). A main reason that limited the potential growth of allografted, and especially xenografted tissue (donor and recipient being from different species), was the lack of widespread availability of proper recipient animal models.

Immunodeficient mice are unable to mount cellular immune reactions against xenografts. This is due to natural or induced mutations causing absence of the thymus (e.g., nude mice) or otherwise genetically rendering T-cells non-functional (e.g., severe combined immunodeficient SCID mice) (Belizario 2009). The use of immunodeficient mice for cross-species transplantation of testicular tissue was reported to only allow early differentiation of germ cells (Skakkebaek et al. 1974, Hochereau-de-Reviers \& Perreau 1997). In 2002, we reported the application of immunodeficient mice as recipients for testis tissue xenografting (TTX) resulting in complete cross-species spermatogenesis (Honaramooz et al. 2002a). In this study, we grafted small fragments of testis tissue (each $\sim 1 \mathrm{~mm}^{3}$ ) from immature mouse, pig or goat donors (1-2 $\mathrm{d}$, 1-wk or 4-wk of age, respectively) into the back skin of castrated immunodeficient nude mice. The subcutaneous grafting site provided an easy access for grafting and observing the graft growth, and its temperature $\left(\sim 36.5^{\circ} \mathrm{C}\right)$ was approximately the same as that in the scrotum of most domestic mammals. Following grafting, not only did the testis fragments increase in size (some up to 100-fold), but mouse gonadotrophins also effectively supported the development, differentiation and complete spermatogenesis of the xenografts. As such, this was the first report of initiation of complete spermatogenesis and steroidogenesis in testis tissue from a different donor species grafted in mice. It was also the first time donor-derived sperm had been produced in testis tissue xenografts originating from neonates. The kinetics of testicular development, somatic and germ cell differentiation, and efficiency of sperm production in pig and goat xenografts were generally similar to the intact testis. Interestingly, elongated spermatids appeared slightly earlier in pig testis xenografts (by about a month) than in age-matched pig testes. The resultant sperm retrieved from the murine, porcine and caprine grafts showed the typical characteristics of testicular sperm of the donor species, and were fertile after intracytoplasmic sperm injection (ICSI) (Honaramooz et al. 2002a).

In the same year, spermatogenesis was also observed after TTX using immature hamsters, monkeys (Schlatt et al. 2002), and rabbits, followed by production of offspring using sperm retrieved from rabbit xenografts (Shinohara et al. 2002). Ever since, TTX has been applied using testis tissue from a range of donor species including rodents, companion animals, livestock, exotic/non-domestic/endangered animals, monkeys and humans; making TTX one of the most versatile and widely applicable models in reproductive science. This article is intended 
to provide a focused review of the salient results of TTX in ruminants, along with its potential applications for the study and manipulation of testis function in various ruminant species.

\section{Rationale and potential applications}

Testis tissue xenografting has the potential to be used in several experimental, applied or clinical applications. For instance, TTX can be used as a model to study testis function, for which few other in vitro or in vivo models exist. Recently, breakthrough in vitro models have been introduced for the production of normal/fertile mouse sperm using neonatal testis cells or tissue in a culture system (Sato et al. 2011, Abu Eljija et al. 2012). Though promising, these in vitro models do not replicate testis function, and are not yet applicable to other species.

A previous in vivo model, referred to as germ cell transplantation, in which isolated testis cells from a fertile donor mouse are microinjected into the seminiferous tubules of infertile recipient mice also initiated and maintained donor-derived spermatogenesis (Brinster \& Zimmermann, 1994). This powerful model quickly became an indispensible functional assay for the study of spermatogonial stem cells (reviewed in Brinster 2007, Honaramooz \& Yang 2011). Subsequently, homologous transplantation of testis cells was extended to other species including ruminant models (Honaramooz et al. 2002b, 2003a, 2003b, Izadyar et al. 2003, Herrid et al. 2006, Rodriguez-Sosa et al. 2006), and surprisingly even cross-species transplantation of isolated testis cells from donor rats and hamsters into recipient mice was also successful in induction of donor-derived spermatogenesis (Clouthier et al. 1996, Ogawa et al. 1999). However, crossspecies transplantation of isolated testis cells from non-rodent species into the seminiferous tubules of immunodeficient mice did not result in complete spermatogenesis, likely as a result of the incompatibility of donor germ cells and recipient's supporting somatic cells (Dobrinski et al. 1999, 2000, Nagano et al. 2001, 2002). Testis tissue xenografting, on the other hand, can be used to study different aspects of testis function of a variety of donor species, including laboratory and domestic/non-domestic animals and even primates (Honaramooz et al. 2002a, 2004, 2005, Schlatt et al. 2002, 2006, Shinohara et al. 2002, Oatley et al. 2004, Snedaker et al. 2004, Rathi et al. 2005, 2006, Arregui et al. 2008a, 2008b, 2013, Abrishami et al. 2010a, Abbasi \& Honaramooz 2011a, 2012, Gourdon \& Travis 2011, Campos-Junior et al. 2014). The key factor in the success of TTX is maintaining the structural integrity of the testis tissue, and allowing the somatic and germ cells to maintain normal interactions (Honaramooz et al. 2002a).

An important advantage of using TTX as a study tool is the accessibility and ease of manipulation of the testis tissue and its environment in the host mouse, something that is not feasible in many donor species. In effect, TTX model uses the laboratory mouse as an in vivo incubator to support the development of xenografted testis tissues. This application will allow, for instance, analysis of the effects of potential toxicants and screening of new drugs or candidate hormonal regimens on testis function, without the use of the target species. For instance, in a hypothetical situation where a research team is testing different treatments to alter age at puberty (e.g., advancing it for dairy or delaying it for beef bull calves), using TTX from calves would allow screening of a large number of candidate treatments in the mouse model, before narrowing the choices to a few promising treatments to be tested on actual bull calves. In such an application, the savings in time and animal costs alone would make the TTX model highly advantageous, because the costs of using mice is considerably less than using herds of cattle.

Furthermore, a unique advantage of using the TTX model is the possibility of subjecting the "same" testis tissue (e.g., literally from one single animal) to different treatments by grafting 
its fragments into multiple recipient mouse groups, and analysing them at different time points. This represents a step forward in investigating the risk assessment of new treatments/ drugs/interventions and their mechanisms of action on testis function without using the target species. Using testes collected at routine castration of, for example, calves to study bovine testis function using TTX into mice, rather than whole animal use of calves, is also consistent with the $3-R$ principle (i.e., replace, reduce and refine) promoted by many institutional committees on ethical use of animals in research. The use of TTX as a means for testing the developmental potential of a given donor testis after manipulation or as a new diagnostic tool in andrology and related research is already underway (Geens et al. 2006, Schlatt et al. 2006, Gertow et al. 2007, Goossens \& Tournaye 2007, Hou et al. 2007, Jahnukainen et al. 2007a, 2007b, Keros et al. 2007, Wyns et al. 2007, 2008, Fujita et al. 2008, Mitchell et al. 2010, Sato et al. 2010).

To expand the application of TTX to field or clinical situations where grafting of freshly collected tissues may not be readily possible or desired, others and we have shown that testicular tissue from several species can be preserved by cooling, hypothermic-preservation, and cryopreservation (both slow-freezing and vitrification), and still maintain its potential for development (discussed in details below, reviewed in Honaramooz 2012). Moreover, others and we showed that the goat, pig and peccary sperm retrieved from the xenografts were fertilisation-competent after ICSI (Honaramooz et al. 2002a, Campos-Junior et al. 2014), capable of supporting development to the blastocyst stage in mice, pigs and rhesus monkeys (Honaramooz et al. 2002a, 2004, 2008), or to term after embryo transfer in mice, rabbits and pigs (Shinohara et al. 2002, Schlatt et al. 2003, Nakai et al. 2010). Combining testicular tissue preservation, TTX, and ICS and other reproductive technologies open the possibility of grafting fresh or preserved testis tissue from immature individuals for production and use of fertile sperm, with important applications. For instance, TTX can provide an alternative genetic rescue strategy for rare/valuable breeds, prized livestock or non-domestic/endangered animals that die before reaching puberty (Arregui et al. 2008a, Abbasi \& Honaramooz 2011a, 2012, Reddy et al. 2012, Arregui et al. 2013, Campos-Junior et al. 2014), or for neonatally lethal phenotypes of experimental, transgenic or cloned animals (Honaramooz et al. 2005, Ohta \& Wakayama 2005, Naughton et al. 2006, Zeng et al. 2011).

Spermatocytes appeared slightly earlier in pig testis xenografts and sperm were seen significantly earlier in rhesus monkey and water buffalo xenografts than in age-matched testes (Honaramooz et al. 2002a, 2004, Reddy et al. 2012). These observations motivate contemplating future application of TTX in farm animal production research aimed at decreasing age at sperm production to reduce generation interval and increase genetic improvement. However, as discussed below, such applications require further optimisation of the TTX model since currently the timing for onset of complete spermatogenesis (appearance of first sperm/elongated spermatids) in xenografts varies greatly depending on the donor species. Without exogenous treatments of the recipient mice, this timing for a given donor type may be advanced, remain the same, or even be delayed compared to testes in situ, and this would have to be determined empirically for each new species. Finally, preliminary results show that TTX may offer a novel method for ex situ production of genetically modified sperm of farm animals by inserting genes of interest into the tissue prior to grafting (Oatley et al. 2004).

\section{Methodology}

Fig. 1 represents the procedures involved in TTX. Donor tissue to be used in TTX is obtained through aseptic removal of the testes (at castration or even shortly after unexpected death of 


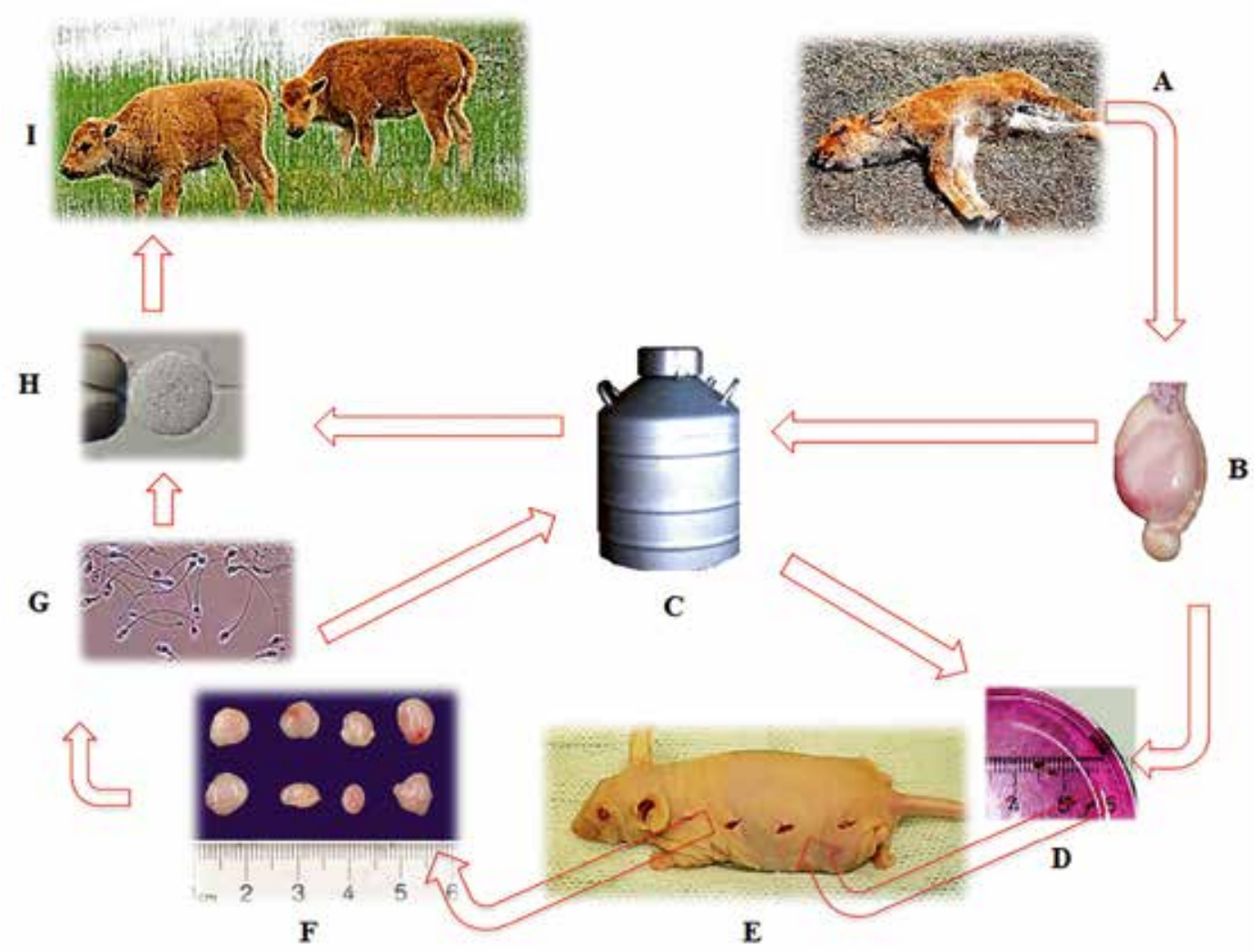

Fig. 1. Schematic overview of different steps in the application of testis tissue xenografting from an immature donor animal into a recipient mouse. The testes are collected from a donor animal (A), which could include post-mortem testis recovery from a recently deceased newborn animal of a valuable/rare/ endangered species. The testis tissue (B) could be cryopreserved (C) until grafting at a later time. At the time of grafting, tissue fragments of $\sim 5 \mathrm{mg}$ are prepared (D) and the fragments are grafted subcutaneously under the back skin of immunodeficient recipient mice (E). When given enough time, the xenografts can grow in size $(\mathrm{F})$ and undergo development, leading to the production of complete spermatogenesis, and fertilisation-competent sperm. The sperm can then be extracted (G) from the grafts and used in intracytoplasmic sperm injection (ICSI) $(\mathrm{H})$, which after embryo transfer can potentially lead to birth of progeny (I). In the absence of technologies for ICSI or embryo transfer for a given species, the fully developed xenografts or their extracted sperm can also be cryopreserved (C) for future use when the required technologies are in place.

a donor animal) and transferred to the laboratory in ice-cold saline. There are indications that the immediate cooling of the tissue is critical to maximize the developmental potential of the tissue (Abbasi \& Honaramooz 2011a). If the recipient mice are not available for immediate TTX of donor tissue from a valuable individual, short-term preservation of the testes should be considered for shipment to a laboratory where immediate TTX or cryopreservation of the tissue is possible (reviewed in Honaramooz 2012). Once in the laboratory, the testes are rinsed multiple times in saline containing antibiotics, and the tunica albuginea, rete testis and overt connective tissue removed. The testis parenchyma is then divided into small sizes of choice (e.g., $\sim 5 \mathrm{mg}$ ) and the fragments maintained in a basic culture media (e.g., Dulbecco's modified 
Eagle's medium, DMEM) on ice until grafting into the recipients, which should be within a few hours. The recipient immunodeficient mice are anesthetised, usually gonadectomised (although apparently not necessary, Abbasi \& Honaramooz 2010, Reddy et al. 2012), and receive multiple transverse linear incisions into the back skin $(5-10 \mathrm{~mm}$ in length, usually up to 4 incisions on each side of the spine). Using blunt dissection, small pockets are created in the subcutaneous fascia, and a fragment of the donor testis tissue is placed subcutaneously inside each pocket. Affixing the fragment by suturing it to the subcutaneous or muscle tissue, as originally performed (Honaramooz et al. 2002a), is not necessary since it may damage the fragments. The incisions are then closed with wound clips (Honaramooz et al. 2002a, Tang et al. 2012). Care should be exercised when cutting, handling or transferring the fragments using fine forceps so as to avoid damaging the tissue by compressing it. Alternatively, cancer implant needles can be used to insert the tissue fragments subcutaneously without a need to make skin incisions (Schlatt et al. 2010).

Since the time taken from the immature state of the tissue to onset of complete spermatogenesis varies among donor species, the post-grafting time-points for graft analysis are planned depending on the donor species. After TTX using immature tissue from most examined species, including calves, certain aspects of testicular development in the grafts may start earlier as compared with age-matched testes. This includes a rapid increase in the number, maturational state and secretion activity of Sertoli cells, causing an accelerated transformation of immature seminiferous cords into seminiferous tubules, which may be followed by earlier appearance of pachytene spermatocytes. However, in most examined species, the onset of complete spermatogenesis (appearance of first sperm/elongated spermatids) tends to be the same time as that for testes in situ. A notable exception is the significant acceleration of sperm production (by several months) seen after TTX from immature rhesus monkeys or water buffalo calves (Honaramooz et al. 2004, Reddy et al. 2012).

After retrieval of the xenografts, they are processed for (immuno)histology and assessed in terms of development, progression of spermatogenesis, general morphology and size of the seminiferous tubules, as compared with the donor tissue at grafting or with age-matched testes. Developmental progression is documented by quantitative evaluation of the tubular morphology and identification of the most advanced germ cell type present in the seminiferous tubules. Furthermore, since vesicular glands are androgen-dependent and regress significantly in the absence of androgens, their weight can be used as an indicator of bioactive testosterone released by the grafts in castrated recipient mice. Serum levels of gonadotropins and testosterone in the host mice can also be measured in the blood taken at the time of sacrifice (Honaramooz et al. 2002a, 2004, Schlatt et al. 2003).

\section{Domestic ruminant donors}

\section{Goats}

As the first ruminant model to be used in TTX, we showed that TTX of testis fragments from 4-wk old goat donors into recipient mice could result in complete spermatogenesis in the grafts within 10 wk (Honaramooz et al. 2002a). Goat testis tissue xenografts underwent testicular maturation, transforming seminiferous cords (with gonocytes as the only type of germ cells present) into seminiferous tubules containing complete spermatogenesis, with first sperm appearing at a time similar to that in testis in situ. Interestingly, the quantity of sperm production in goat testis tissue xenografts $\left(67 \times 10^{6}\right.$ sperm per gram tissue) was comparable to that of testes in situ, and the resultant sperm were fertile after ICSI in a mouse oocyte assay (Honaramooz et al. 2002a). 
Unlike the promising results from immature donor goats, when we used adult donor goats (1.5- or 4-year old), TTX was not successful and led to degeneration of the seminiferous tubules or the entire grafts (Arregui et al. 2008a); however this was not unique to mature donor goats and TTX from mature testis tissue of other species also showed significantly diminished developmental potential. During the grafting procedures and immediately afterward, all testis tissue fragments undergo some degree of damage due to hypoxia and ischemia; however, the immature tissue has better ability to recover from the damage (Schlatt et al. 2002, Rathi et al. 2006, Abrishami et al. 2010b). The inability of the adult testis tissue to thrive after xenografting has been attributed to the high sensitivity of differentiated germ cells to ischemia, reduced ability of the tissue for neo-angiogenesis, and inability of mature Sertoli cells to regenerate (Schlatt et al. 2002, Meachem et al. 2005, Arregui et al. 2008b, Schmidt et al. 2007, Abrishami et al. 2010a). Therefore, testis tissue xenografts from newborn and prepubertal donors overall have higher rates of survival, cell differentiation, and spermatogenic support than the donor tissues with pre-existing spermatogenesis at the time of grafting (Huang et al. 2008, Abrishami et al. 2010a).

\section{Sheep}

Using neonatal and prepubertal lambs as testis tissue donors, others and we observed completion of ovine spermatogenesis after TTX into host mice (Zeng et al. 2006, Arregui et al. 2008a, Rodriguez-Sosa et al. 2010). We also reported that first elongated spermatids, as evidence of complete spermatogenesis, appeared at the same time in grafts and in age-matched sheep (i.e., $\sim 12$ wk post-grafting) (Arregui et al. 2008a). High percentage of grafted fragments developed into visible grafts (78-95\%, Zeng et al. 2006, Arregui et al. 2008a), and interestingly, an average of $64 \%$ of seminiferous tubules in grafts of neonatal lamb donors examined 6-7 months postgrafting contained sperm (Zeng et al. 2006). This made ovine grafts one of the most efficient in terms of spermatogenic activity among testis grafts from both ruminant and non-ruminant species. The length of each spermatogenic cycle in ovine grafts was also shown to be similar to that in situ (Zeng et al. 2006). We also demonstrated that individual cells obtained after complete enzymatic dissociation of neonatal lamb testis tissue, once transferred under the skin of recipient nude mice, can undergo extensive rearrangement to form functional testicular tissues with complete spermatogenesis, at times indistinguishable from the intact testis tissue (Arregui et al. 2008a). Although not TTX per se, this latter experiment showed that transplantation of testis cell aggregates can provide an excellent model for the study of cellular migration and testis organogenesis using different animal models (Gassei et al. 2006, Honaramooz et al. 2007, Arregui et al. 2008a).

\section{Cattle}

Most of the literature on TTX using ruminant donors has focused on cattle, partly because of its importance as a ruminant species, but also because of its unique challenges including the slow progression and low efficiency of spermatogenesis in grafts from bovine donors (Oatley et al. 2004, Rathi et al. 2005, Schmidt et al. 2006a, Huang et al. 2008), as compared with sheep and goats (Honaramooz et al. 2002a, Zeng et al. 2006, Arregui et al. 2008a, 2008b, Rodriguez-Sosa et al. 2010). Nevertheless, TTX of immature bovine donors into recipient mice resulted in moderate graft recovery rates and a slow (24-32 wk) and inefficient (in $<15 \%$ of seminiferous tubules), yet eventual completion of spermatogenesis (reviewed in RodriguezSosa \& Dobrinski 2009). 
When prepubertal Holstein bulls of 6 different ages (12 to $32 \mathrm{wk}$ ) were used as donors in TTX, the presence of more advanced germ cells in the donor tissue at the time of grafting was correlated with poor post-grafting testicular development and differentiation. Conversely, the abundance of undifferentiated germ cells at the time of grafting resulted in better graft development (Huang et al. 2008). In this latter study, the spermatogenic efficiency of all examined prepubertal ages, especially for $>20 \mathrm{wk}$ old donors, was very low $(<3 \%)$, while seminiferous tubular degeneration was very high ( $>70 \%)$. It was also concluded that regardless of the donor age, the timing for restoration of spermatogenesis in grafts seemed to remain constant, indicating that spermatogenesis after grafting needed to be (re)established from the spermatogonial stage (Huang et al. 2008). This lack of thriving by testis grafts of prepubertal bulls perhaps was not surprising, given that others and we have documented the adverse effects of advancing age on TTX outcomes in multiple species (Kim et al. 2007, Arregui et al. 2008b, Abrishami et al. 2010a). However, it was rather surprising that the potential for graft growth and development also significantly varied even among early postnatal calves (1, 2, 4 and 8 wk of age; Oatley et al. 2005, Schmidt et al. 2006a). Donor tissue from both 1- and 8-wk old bull calves had higher spermatogenic efficiency (\% of tubules with sperm/elongated spermatids) than 2- or 4-wk olds, while testis tissue from 1- or 2-wk old calves had the greatest potential for growth (increase in graft size) (Oatley et al. 2005, Schmidt et al. 2006a). The authors then followed up their observations by analysing differential gene expression in the donor tissues of early postnatal ages (2-, 4- and 8-wk olds) as well as during the grafting period (Schmidt et al. 2007). It was concluded that lowered Sertoli cell-specific gene expression in 8-wk old donor tissue grafts might have contributed to prevention of over-proliferation of Sertoli cells, which otherwise would have resulted in increases in intratubular pressure deemed to be detrimental to germ cells. Additionally, lower expression of the germ cell-specific gene KIT was observed in all testis grafts, which may partly explain the compromised initiation of germ cell differentiation in the grafts. When testes of calves were compared at 2, 4, or 8 wk of age, several genes involved in angiogenesis or tissue growth were lower in testes of relatively older calves (Schmidt et al. 2007). This conclusion was consistent with an earlier observation where supplying bovine testis tissue fragments with vascular endothelial growth factor (VEGF) at the time of grafting led to both increased physical growth and spermatogenic efficiency of grafts collected 6 months later (Schmidt et al. 2006b).

There are conflicting reports on the timing for onset of complete spermatogenesis in bovine testis tissue xenografts, with one study using 4-wk old donor calves (of unspecified breed) showing a similar rate to testes in situ (Oatley et al. 2004), while another report from the same group using Angus-cross calves of different ages showed slight acceleration of this timing only for 1-wk old donors (Schmidt et al. 2006a). However, in addition to differences in donor ages between the two latter studies, the results from beef calf grafts were compared with the available literature on timing of germ cell development in Holstein calf testes, which may have contributed to the reported discrepancies. We used 1-2-wk old Holstein bull calves as donors for TTX and compared the results to testes of control Holstein calves castrated at times corresponding to the cumulative age of the grafts (Rathi et al. 2005). We observed a rapid rate of post-grafting increase in the seminiferous tubule lumen formation, an initial reduction in germ cell numbers (by 2-mo post-grafting vs. an increase in controls), earlier appearance of first pachytene spermatocytes in grafts (before 4-mo post-grafting vs. 5-mo age in controls). There was a developmental pause at the pachytene spermatocyte stage in most grafts, but appearance of first elongated spermatids in grafts occurred at a time similar to controls (6- to 8-mo), although in much lower numbers (Rathi et al. 2005). In a study of TTX using testis tissue collected from mid-gestation bovine fetuses, it was demonstrated that fetal tissue had partial potential for development and underwent tubular 
expansion, androgen production, and differentiation up to the spermatocyte stage in samples taken at 10 mo post-grafting (Rodriguez-Sosa et al. 2011). Given the expectations raised by the very high developmental potential of neonatal testis tissues after xenografting, the results of TTX using bovine fetal donors may be viewed as somewhat underwhelming. Nevertheless, these and similar results point to yet another application of TTX: i.e., as a model to study fetal testicular development from diverse species (Yu et al. 2006, Mitchell et al. 2010, Sato et al. 2010, Rodriguez-Sosa et al. 2011).

\section{Exotic, rare or endangered ruminant donors}

Currently, at least 140 species of bovidae and 55 species of cervidae family are thought to be under some degree of threat (IUCN 2013, www.iucnredlist.org). Although preservation of the habitant remains the first priority for reducing the speed of decline of vulnerable species, assisted reproductive technologies (ART) such as breeding and spreading the species ex situ may be applicable for some species. Conservation of rare or endangered species through rescuing and archiving of the germline and genetic diversity is probably one of the most challenging responsibilities of conservation and reproductive biologists/technologists (reviewed in Andrabi \& Maxwell 2007). Although procedures are in place for retrieval and cryopreservation of ejaculated, epididymal or testicular sperm, even shortly after death of an adult animal, they are not applicable to sexually immature males. The use of TTX provides a previously unavailable system for conservation purposes to produce sperm from valuable immature animals that die before reaching puberty.

As a first attempt to salvage the genetic material and fertility potential of a rare, valuable animal, we used TTX from a newborn Javan banteng (Bos javonicus) calf into recipient mice. The calf that died shortly after birth was cloned from donor cells of a genetically valuable banteng frozen some 25 years earlier. Although pachytene spermatocytes appeared in the banteng xenografts as early as 3 mo after grafting, spermatogenesis did not proceed further through meiosis in grafts analysed 6, 9, 12 or 15 mo after grafting, although xenografts were capable of releasing bioactive testosterone. These results indicated that banteng spermatogenesis was initiated in the mouse host but became arrested at meiosis; therefore, haploid germ cells could not be recovered (Honaramooz et al. 2005). This observation also underscored that the efficiency of sperm production in testis xenografts for new species may be unpredictable and has to be determined empirically for different donor species.

We later expanded the application of TTX into specialised hoofstock by using testes collected post-mortem from two immature plains bison calves (Bison bison bison) and a white-tailed deer fawn (Odocoileus virginianus), as potential models for closely related rare or endangered bovidae and cervidae species (Abbasi \& Honaramooz 2011a, 2012). In case of bison calf donors, $69 \%$ of the grafted testis fragments were visible as xenografts, growing in weight to $\sim 4$-fold by 2 mo and 10 -fold by 16 mo post-grafting. In bison testis xenografts, gradual maturational changes included first seminiferous tubule expansion by $2 \mathrm{mo}$, spermatocytes by $6 \mathrm{mo}$, round spermatids by $12 \mathrm{mo}$, and elongated spermatids by 16 mo post-grafting. In terms of spermatogenic efficiency, compared to grafts from dairy/beef calves, a smaller number of seminiferous tubules in bison grafts contained complete spermatogenesis $(\sim 1 \%)$ at the latest time points, and the process was further delayed by several months (Abbasi \& Honaramooz 2011a). For white-tailed deer donor, 63\% of the grafted testis fragments were recovered as xenografts and showed a gradual testicular development, starting with tubular expansion by 2 mo, presence of spermatocytes by $6 \mathrm{mo}$, round and elongated spermatids by 8 mo, followed by 
fully-formed sperm by 12 mo post-grafting. The spermatogenic efficiency of deer testis xenografts was higher than that in bison grafts but still low; tubules containing sperm/elongated spermatids accounted for $\sim 8 \%$ of all tubules at the latest time points (Abbasi \& Honaramooz 2012). There were no previous data on the kinetics of germ cell development or testicular maturation for bison or white-tailed deer in situ; however, time at onset of complete spermatogenesis in bison and deer testis xenografts generally corresponded to the reported timing of sexual maturation in each species (Abbasi \& Honaramooz 2011a, 2012). These latter studies demonstrated, for the first time, that TTX from immature bison and deer donors into recipient mice could successfully result in testicular maturation and development of spermatogenesis in the grafts up to the stage of sperm production.

Using TTX from 8-wk old Murrah water buffalo calves (Bubalus bubalis) resulted in a surprisingly early appearance of elongated spermatids 24 wk post-grafting, while they are normally observed in the buffalo testes in situ after 72-84 wk of age. It was also concluded that both spermatogenic efficiency and testosterone production of the xenografts from water buffalos were much lower than that reported for xenografts of bovine testis tissue. Interestingly, acceleration in onset of complete spermatogenesis was only seen if TTX recipient mice were intact, but not when they were castrated (Reddy et al. 2012).

In fact, there may be a common mechanism for both the exceptional acceleration in onset of complete spermatogenesis in xenografts of water buffalo calves ( $\sim 6$ mo for grafts $v s$. $>17$ mo for control testes), and the unusually long pause in meiotic development of xenografts from the banteng calf (even up to 15 mo post-grafting; Honaramooz et al. 2005). The key in success of TTX using water buffalo donors was attributed to the use of intact (non-castrated) nude recipient mice; supported by their observation that TTX from the same water buffalo donors into castrated nude mice failed to develop beyond the spermatocyte stage (Reddy et al. 2012). However, the degree to which the gonadal status of recipient mice affects the results of TTX is controversial, and in a study of dairy calf testis xenografts no differences were noticed between castrated and intact recipients (Huang et al. 2008). After a comprehensive comparison using 8 different recipient models, we also did not see a difference in TTX results between castrated and non-castrated nude mice (Abbasi \& Honaramooz 2010). Reddy et al. (2012) reported a very low androgen releasing activity by the xenografts from water buffalo calves (as compared to the previously reported levels released by the testis xenografts from cattle). This led the authors to speculate that testosterone released by the recipient testes has stimulated the development of the grafted immature buffalo testis tissue, citing the naturally much lower serum levels of testosterone seen in mature water buffalos compared to levels reported in mice (0.6 vs. 2-5 $\mathrm{ng} / \mathrm{ml}$, respectively, Reddy et al. 2012). If the presence of mouse testes is indeed crucial for development of testis xenografts from water buffalo calves, it would be a rather unique effect for water buffalo and possibly certain other donor species. However, considering the similarities and differences between these two latter exceptional models (developmental pause after TTX from both banteng and water buffalos in castrated nude recipients vs. developmental acceleration after TTX from water buffalos in intact recipients), such a theory is plausible, and may lead to speculation that further work in banteng testis xenografts may benefit from grafting into intact recipients or testosterone supplementation of recipients.

Recently, TTX was also applied in two endangered ruminant species using testes collected post-mortem from two Cuvier's gazelle fetuses (Gazella cuvieri) and one 8-mo old Mohor gazelle (G. dama mhorr). It was reported that progression of spermatogenesis was donor age dependent; testis tissue xenografts from Cuvier's gazelle fetuses contained spermatocytes $40 \mathrm{wk}$ post-grafting, and those of 8-mo old Mohor gazelle had round spermatids $28 \mathrm{wk}$ after grafting (Arregui et al. 2013). 


\section{Current challenges and sources of variation}

Since the first successful reports of TTX in 2002, this system has been extensively applied in different settings and often with different perspectives, creating a wealth of new information on the developmental potential and function of testis tissue in a wide range of donors. In order to expand and maximize the benefits of TTX model, there is a continued need for further proof-ofprinciple studies, such as those exploring TTX in new species or its value for new applications. There is also an equally important need for focused research on previously existing and newly discovered challenging aspects of TTX.

As expected, time to maturity and rate of spermatogenic efficiency of TTX vary depending on the species, age or developmental status of tissue donors. However, some of the TTX outcomes also varied when using seemingly similar donor types/conditions (Rathi et al. 2005, 2006, Zeng et al. 2006, Abrishami et al. 2010b). To optimise the TTX model for the study of testis function or production of sperm, different aspects of the model need to be thoroughly evaluated. Therefore, one of the current challenges of TTX, especially from bovine donors, is deciphering all sources of variation in results, which can generally be classified into factors related to the donor type or recipient mouse and are discussed below. There is also a need for further investigation into the underlying mechanisms of known sources of variation and, perhaps more importantly, identifying other causes of variation that cannot be explained by known factors. Other challenging aspects of TTX using ruminant donors include optimising preservation/banking of the tissue prior to TTX, and using the resultant xenogeneic sperm in ART for diverse ruminant species.

\section{Donor factors}

A phylogenetically wide range of donor species has been used for TTX, albeit with speciesspecific differences. In nearly all cases, when immature testis tissue xenografts were allowed sufficient time to develop in the host mice, testicular maturation and completion of spermatogenesis were eventually observed (reviewed in Paris \& Schlatt 2007, Mota et al. 2011, Rodriguez-Sosa \& Dobrinski 2009). Time required for most testis tissue xenografts to develop from an immature state to a fully matured tissue generally correlated with the timing for testes of each species in situ. This timing, for instance, was as early as 3 mo for grafts from lambs, 6-8 mo for dairy/beef calves, 12 mo for white-tailed deer fawn, and > 16 mo for bison calves; nevertheless, it was approximately similar to that in age-matched testes (Rodriguez-Sosa \& Dobrinski 2009, Abrishami et al. 2010a, Abbasi \& Honaramooz 2011a, 2012). Of course, the two prime exceptions to this general rule were the cases of xenografts from banteng and water buffalo calves (Honaramooz et al. 2005, Reddy et al. 2012), which as discussed above deserve further investigation to elucidate the underlying mechanisms.

An important measure of TTX outcome that tends to be overlooked is the efficiency of spermatogenesis; expressed as the prevalence of seminiferous tubules containing sperm/ elongated spermatids in grafts collected at later time points corresponding to the expected maturation of control testes in situ. While the spermatogenic efficiency was high $(>60 \%)$ in testis tissue xenografts from immature goat and sheep donors, allowing the retrieval of high numbers of sperm from the goat xenografts (Honaramooz et al. 2002a, Arregui et al. 2008a), it was low in xenografts from dairy/beef bull $(<15 \%)$, water buffalo $(<3 \%)$, and bison calves $(\sim 1 \%)$ and white-tailed deer ( 8\%) (Oatley et al. 2004, 2005, Rathi et al. 2005, Schmidt et al. 2006a, 2006b, Abbasi \& Honaramooz 2011a, 2012, Reddy et al. 2012). Factors such as differential expression of certain genes involved in germ cell differentiation of bovine xenografts 
have been identified as a likely reason (Schmidt et al. 2007), but there is potential for hormonal interventions of grafts or recipients to slightly alter this default timing of maturation and spermatogenic efficiency (reviewed in Rodriguez-Sosa \& Dobrinski 2009). Therefore, while TTX is applicable for diverse species, the timing of testicular development and efficiency of sperm production in testis xenografts appear to be variable, and need to be empirically determined for new donor species.

The age or developmental status of testis donors at the time of grafting is also a widely studied and important factor affecting the efficiency and outcomes of TTX. Using TTX of a wide range of donor ages in multiple species including sheep, goats, and dairy/beef calves it has been shown that overall immature animals possess a considerably higher potential for successful development than mature donors (Oatley et al. 2005, Arregui et al. 2008b, Huang et al. 2008, Abrishami et al. 2010a). Even among immature testis tissue donors, TTX results may differ depending on seemingly small differences in age or developmental status (Oatley et al. 2005, Schmidt et al. 2006a). Gene expression analysis of the grafts from beef calf donors confirmed that changes in several factors involved in tissue survival, somatic cell function, and germ cell differentiation play a role in determining the developmental potential of grafts from a given age (Schmidt et al. 2007). Further research using these and similar information is needed to elucidate other underlying mechanisms of variation in TTX results, which may also help in designing strategies to support the xenografts or their environment to maximize the efficiency of TTX.

\section{Recipient factors}

Most recent studies using TTX have followed the procedures we described in 2002, in which castrated male nude mice were used as recipients of up to 8 testis fragments in each mouse (Honaramooz et al. 2002a). Castration was generally thought to be vital, or at least helpful, for the success of TTX, and a few indirect comparisons were made between nude and SCID recipients, but no conclusive differences were found in TTX results (Rathi et al. 2005, 2006, Geens et al. 2006). However, no systematic study had investigated the virtue of recipient mouse type for TTX or the optimum number of the initial tissue fragments. We therefore examined the effects of factors related to the choice of recipient immunodeficient mice, including the strain (SCID vs. nude), gender (male vs. female), and gonadal status (intact $v s$. gonadectomised) using a 2X2X2 factorial design (i.e., a combination of eight recipient models, Abbasi \& Honaramooz 2010). Although the overall graft recovery was high $(\sim 94 \%)$, there were some interesting differences among these recipient models. For instance, the grafts recovered from male SCID mice were the heaviest ( $\sim 4$-fold $v s$. grafts from female recipients), and had the highest spermatogenic efficiency among all recipient groups. Equally important, gonadectomy of male or female recipients did not affect the results of TTX. Interestingly, female recipient mice, even those with intact ovaries, were fully capable of providing a supportive milieu for the developing testis tissue xenografts (Abbasi \& Honaramooz 2010).

We also investigated the effect of the initial number of testis tissue fragments on TTX results by grafting 2, 4, 8, or 16 fragments (each $\sim 5 \mathrm{mg}$ ) per castrated recipient mouse (Abbasi \& Honaramooz $2011 \mathrm{~b})$. Although the rate of graft recovery was high in all groups $(86 \%-98 \%)$, not only were the total graft weights much higher (by $\sim 12$-fold) in mice receiving $16 \mathrm{vs} .2$ fragments, but more importantly the average graft weights were also greater $(\sim 2.5$-fold $)$. The spermatogenic efficiency and androgen release were also higher in grafts from mice with 16 vs. 2 fragments. We therefore showed that the recipient mouse is fully capable of supporting the development of a much larger mass of grafted donor testis tissue than routinely used, 
which can maximize the mass of harvested grafts (Abbasi \& Honaramooz 2011b). It should be emphasised that we used porcine testis tissue in these latter studies, which may not necessarily reflect every donor species including ruminants. For example, no differences in TTX results were seen between castrated and intact recipients when using dairy calf testis grafts (Huang et al. 2008), but using intact recipients was reported to be critical for the success of TTX using water buffalo donors (Reddy et al. 2012). Nevertheless, these results add the recipient model and the number of fragments grafted to the list of potential sources of variation in TTX results.

Using TTX as a tool for endocrinology studies has allowed, for example, altering the levels of growth hormones, testosterone or thyroid hormones in recipient mice carrying testis tissue grafts from ruminant donors, and generating new insights on their potential effects on testis function for each species (Rathi et al. 2005, Huang et al. 2008, Rodriguez-Sosa et al. 2012, Reddy et al. 2012). While supplementing exogenous gonadotrophins to the recipient mice after TTX has produced mixed results using non-ruminant donors (Honaramooz et al. 2004, Rathi et al. 2006, 2008, Schlatt et al. 2010, Ehmcke et al. 2011, Van Saen et al. 2013), similar experiments are lacking for ruminant donors.

Considerable incremental progress has been made in multiple areas related to the success rate of TTX resulting, for instance, in increasing the graft recovery rates from an earlier $\sim 60 \%$ (Honaramooz et al. 2002a) to more recent $\sim 95 \%$ using the same neonatal porcine model (Abbasi \& Honaramooz 2010, 2011b). However, further improvement of TTX outcomes through systematic studies for proper selection and supportive hormonal supplementation of recipients are manageable challenges for future research.

\section{Pre-grafting preservation of testis tissue}

Prior to 2002, preservation of testis tissue was largely limited to cooling or freezing of testis biopsies from adult men for later use in testicular sperm extraction and ICSI. However, cryopreservation of immature testis tissue was not widely considered, perhaps due to lack of its foreseen potential applications (reviewed in Orwig \& Schlatt 2005). This view changed when others and we showed for the first time that cryopreservation of immature testis tissue prior to TTX can be done so as to maintain its potential for development (Honaramooz et al. 2002a, Schlatt et al. 2002, Shinohara et al. 2002). Although the efficiency of the cryopreservation protocols used in these earlier studies was low, this proof-of-principle step was crucial in extending the application of TTX model to clinical or field situations, where grafting of freshly collected tissue may not be readily possible or desired. In a relatively short period of time since then, advances in cryopreservation of testicular tissue have opened new possibilities for preservation of male fertility from immature individuals (Schlatt et al. 2006, Jahnukainen et al. 2007a, Keros et al. 2007, Wyns et al. 2007, 2008, Zeng et al. 2009, Abrishami et al. 2010b).

As reviewed elsewhere (Honaramooz 2012), most studies on cryopreservation of testis tissue have used a mouse, pig or primate model, and not ruminant donors. We now know that the testis tissue from each donor species/age may respond differently to a given cryopreservation protocol, and in order to maximize the tissue integrity, viability and subsequent post-grafting functional competence, several variables may need to be examined. These differences may be related to differing testicular architecture (\% of fibrotic tissue), morphology, or lipid composition. In fact, optimal cryopreservation for a given tissue requires examination and refinement of freezing and thawing rates, osmotic conditions, choice and concentration of cryoprotectants, and equilibration times in cryoprotective solutions (Honaramooz 2012). Nevertheless, as we have shown after comparing several strategies using a neonatal porcine model, it is possible to properly preserve the donor testis tissue using cooling (for up to 3 days), hypothermic 
preservation (for up to 6 days), slow/controlled freezing, or vitrification protocols (for extended period of times) prior to TTX, to achieve results that are nearly as high as using fresh tissues (Abrishami et al. 2010b, Yang et al. 2010). Our successful vitrification of immature testis tissue was a first and made it considerably easier to freeze testis tissue pieces in a farm or small clinic settings, because vitrification does not require the extensive laboratory equipment commonly used for programmed slow freezing (Abrishami et al. 2010b).

In addition to its experimental value, preservation of testis tissue has applications in both reproductive medicine and animal conservation. These include preserving the fertility potential of prepubertal boys undergoing gonadotoxic cancer therapies, and salvaging the genetic potential of immature endangered animals or valuable breeds of livestock through banking of gonadal tissue (reviewed in Honaramooz 2012). Efforts to improve post-preservation efficiency of testis tissue are needed in all these important areas, and especially are lacking for ruminant species.

\section{Subsequent use of graft-derived sperm}

Morphologically normal and viable sperm have been observed in testis tissue xenografts from several species and, after mechanical dissociation, these xenogeneic sperm could be retrieved at high concentrations. Since the grafts develop from small fragments of testicular parenchyma, the resultant sperm do not undergo epididymal maturation, making them comparable to testicular sperm in their maturation status. Behaving as testicular sperm, graft-derived sperm are immotile and may only gain weak mobility after dilution in manipulation media, and therefore, would have to be used in ICSI. Following ICSI in multiple species, the capacity of graft-derived sperm for fertilising oocytes (Honaramooz et al. 2002a, Campos-Junior et al. 2014), driving early embryonic development (Honaramooz et al. 2002a, 2004, 2008) leading to the birth of healthy offspring have been shown (Shinohara et al. 2002, Schlatt et al. 2003, Nakai et al. 2010). An option is available to infertile men with non-sperm differentiated germ in their testis biopsies, where elongated spermatids, or even round spermatids can be extracted and used for fertilisation of oocytes through ICSI (reviewed in Vloeberghs et al. 2013). It remains to be seen if similar microinjection techniques can be developed for situations where only non-sperm germ cells are developed in grafts.

It is important to note that using the xenogeneic sperm to produce offspring is currently limited to species in which the full complement of subsequent ART is already well established. These ART are species-specific and require sufficient knowledge of the reproductive biology of both males and females of the target species. For example, for the successful production of offspring from a given species several procedures are required including estrous cycle synchronisation, efficient collection of mature oocytes, performing ICSI, culturing early embryos, preparing surrogate females, and successful transfer of the embryos to the surrogate females. These procedures are routinely done in only a handful of species (reviewed in Andrabi \& Maxwell 2007). Therefore, a major challenge ahead for the practical use of xenogeneic sperm from many species is the development of the required ART for each species, which in turn requires a working knowledge of the reproductive physiology and management of the target species.

However, it is also important to note that the lack of subsequent ART available for using xenogeneic sperm from a given species should not diminish the value of TTX as a unique method to produce sperm from even a recently dead neonatal individual from that same species. This is because one may cryopreserve the donor tissue prior to grafting, or proceed with TTX and freeze the matured grafts or extracted sperm until such time when the required subsequent ART do become available for the target species (Fig. 1). Furthermore, TTX has multiple immediate 
applications, which do not rely on other technologies, including its use as a tool for the study of testis function of the donor species. Lastly, aside from TTX, no feasible alternatives are currently available to preserve the genetics/fertility of immature valuable animals. In vitro production of sperm from neonates has so far been only achieved in mice (Sato et al. 2011, Abu Eljija et al. 2012). Cloning has been used for a number of species and can be useful especially if the goal is to produce a genetically exact replica of an individual animal. Development of cloning for a new species is also costly, time consuming, and technically demanding; hence, successful production of healthy cloned offspring is limited to handful of economically important domestic animal species. Perhaps due to a lack of sufficient commercial or research interest, little progress has been made in cloning technology for non-domestic and endangered species (reviewed in Mastromonaco \& King 2007). Furthermore, in animal conservation, it is important to preserve the potential contribution to the genetic variability of as many members of a rare or endangered species/breed as possible, while cloning does not immediately provide the genetic diversity that would otherwise be offered by gametes.

\section{Conclusions}

Xenografting of testis tissue has great potential as a valuable in vivo culture system to mimic testis development and spermatogenesis of a variety of different species ex situ. It offers a unique approach for the study or manipulation of testis function of the target species, with the flexibility and convenience of working with a laboratory mouse in which the hormonal milieu can be more easily manipulated. Multiple studies have used TTX from donors of domestic and exotic/ non-domestic animals, and shown that once the grafts are allowed sufficient time to develop, complete donor-derived spermatogenesis can be expected. Nonetheless, testis tissue from each donor species/developmental status displayed some universal and some unique patterns, leading to conclusions about the superior potential of immature testis tissue as donors and the species-specific timing of testis development and spermatogenic efficiency.

As a general rule, while for most donor species the initial expansion of seminiferous tubules in grafts may happen earlier, the appearance of first sperm/elongated spermatids is usually observed at the same time as in the age-matched testes; however, in certain species/breeds it may be slightly earlier or even delayed. For instance, after TTX using dairy/beef calf donors, the unexpected observations of a pause in meiosis progression led to testing of new hypotheses, resulting in novel discoveries about the genetic and hormonal regulation of bovine testis development. Sperm recovered from testis tissue xenografts when used in ICSI can fertilise oocytes and support development to term. Using TTX for every new species has also brought considerable new knowledge about the donors' testis biology, including some reports, which were the first examinations of testes for a given species; hence, no comparable in situ data were available to compare with xenografts results. Post-mortem recovery of testes and use in TTX after unexpected demise of immature calves/fawns, exemplified its potential application for rare or endangered ruminant species. Owing to TTX, cryopreservation of immature testis tissue now is being considered for preserving the fertility potential of prepubertal boys undergoing gonadotoxic cancer therapies, and salvaging the genetic potential of immature endangered animals or valuable breeds of livestock.

Systemic evaluations of multiple factors related to the success rate of TTX, such as, the choice and hormonal status of recipients, sources of variation, and their underlying mechanisms, will further improve the outcomes of TTX. Additional research focusing on optimisation of cryopreservation protocols for testicular tissue to salvage fertility potential of immature 
individuals will result in more widespread application of TTX technology. The use of graftoriginated sperm for fertilisation and production of progeny requires the availability of ICSI and complementary ART for the target species. In cases, for example, where a male offspring of an endangered species dies before producing sperm, it may be necessary to consider pregrafting cryopreservation of the donor testicular tissue or post-grafting freezing of the xenografts or graft-derived sperm until the required ART is developed. While overcoming some of these cited challenges is achievable in a foreseeable future, the development of ICSI and subsequent ART for new, especially non-domestic, species requires significant time, capital and a collective effort by conservation and reproductive scientists from different fields to overcome, and may remain challenging into the more distant future.

\section{Acknowledgements}

The author would like to thank the current and former members of the laboratory for their diligent work cited here, the Natural Sciences and Engineering Research Council (NSERC) of Canada, and the Saskatchewan Health Research Foundation (SHRF) for grants to support the recent research.

\section{References}

Abbasi S \& Honaramooz A 2010 Effects of recipient mouse strain, sex and gonadal status on the outcome of testis tissue xenografting. Reproduction, Fertility, and Development 22 1279-1286.

Abbasi S \& Honaramooz A 2011a Xenografting of testis tissue from bison calf donors into recipient mice as a strategy for salvaging genetic material. Theriogenology 76 607-614.

Abbasi S \& Honaramooz A 2011b The number of grafted fragments affects the outcome of testis tissue xenografting from piglets into recipient mice. Veterinary Medicine International 686570 1-7.

Abbasi S \& Honaramooz A 2012 Feasibility of salvaging genetic potential of post-mortem fawns: production of sperm in testis tissue xenografts from immature donor white-tailed deer (Odocoileus virginianus) in recipient mice. Animal Reproduction Science 135 47-52.

Abrishami M, Abbasi S \& Honaramooz A 2010a The effect of donor age on progression of spermatogenesis in canine testicular tissue after xenografting into immunodeficient mice. Theriogenology 73 512-522.

Abrishami M, Anzar M, Yang Y \& Honaramooz A 2010b Cryopreservation of immature porcine testis tissue to maintain its developmental potential after xenografting into recipient mice. Theriogenology 73 86-96.

Abu Elhija M, Lunenfeld E, Schlatt S \& Huleihel M 2012 Differentiation of murine male germ cells to spermatozoa in a soft agar culture system. Asian Journal of Andrology 14 285-293.

Andrabi SM \& Maxwell WM 2007 A review on reproductive biotechnologies for conservation of endangered mammalian species. Animal Reproduction Science $\mathbf{9 9}$ 223-243.

Arregui L, Rathi R, Megee SO, Honaramooz A, Gomendio M, Roldan E \& Dobrinski I 2008a Xenografting of sheep testis tissue and isolated cells as a model for preservation of genetic material from endangered ungulates. Reproduction 136 85-93.

Arregui L, Rathi R, Zeng W, Honaramooz A, Gomendio M, Roldan ERS \& Dobrinski I 2008b Xenografting of adult mammalian testis tissue. Animal Reproduction Science 106 65-76.

Arregui L, Dobrinski I \& Roldan ER 2013 Germ cell survival and differentiation after xenotransplantation of testis tissue from three endangered species: iberian lynx (Lynx pardinus), Cuvier. Reproduction, Fertility, and Development doi: 10.1071/RD12411.

Belizario JE 2009 Immunodeficient mouse models: an overview. The Open Immunology Journal 2 79-85.

Brinster RL 2007 Male germline stem cells: from mice to men. Science 316 404-405.

Brinster RL \& Zimmermann JW 1994 Spermatogenesis following male germ-cell transplantation. Proceeding of the National Academy of Science USA 91 11298-11302.

Campos-Junior PH, Costa GM, Avelar GF, Lacerda SM, da Costa NN, Ohashi OM, Miranda Mdos S, Barcelos LS, Jorge EC, Guimarães DA \& de França LR 2014 Derivation of sperm from xenografted testis cells and tissues of the peccary (Tayassu tajacu) Reproduction 147 291-299.

Clouthier DE, Avarbock MR, Maika SD, Hammer RE \& Brinster RL 1996 Rat spermatogenesis in mouse testis Nature 381 418-421.

Deanesly R 1954 Spermatogenesis and endocrine activity in grafts of frozen and thawed rat testis. Journal of Endocrinology 11 201-206.

Dobrinski I, Avarbock MR \& Brinster RL 1999 Transplantation of germ cells from rabbit and dogs into mouse testes. Biology of Reproduction 61 1331-1339.

Dobrinski I, Avarbock MR \& Brinster RL 2000 Germ cell 
transplantation from large domestic animals into mouse testes. Molecular Reproduction and Development $\mathbf{5 7}$ 270-279.

Ehmcke J, Gassei K, Westernstroer B \& Schlatt S 2011 Immature rhesus monkey (Macaca mulatta) testis xenografts show increased growth, but not enhanced seminiferous differentiation, under human chorionic gonadotropin treatment of nude mouse recipients. International Journal of Andrology 34 459-467.

Fujita K, Tsujimura A, Hirai T, Ohta H, Matsuoka Y, Miyagawa Y, Takao T, Takada S, Nonomura N \& Okuyama A 2008 Effect of human leukemia cells in testicular tissues grafted into immunodeficient mice. International Journal of Urology 15 733-738.

Gassei K, Schlatt S \& Ehmcke J 2006 De novo morphogenesis of seminiferous tubules from dissociated immature rat testicular cells in xenografts. Journal of Andrology 27 611-618.

Geens M, de Block G, Goossens E, Frederickx V, van Steirteghem A \& Tournaye H 2006 Spermatogonial survival after grafting human testicular tissue to immunodeficient mice. Human Reproduction 21 390-396.

Gertow K, Przyborski S, Loring JF, Auerbach JM, Epifano O, Otonkoski T, Damjanov I \& Ahrlund-Richter L 2007 Isolation of human embryonic stem cell-derived teratomas for the assessment of pluripotency. In Current Protocols in Stem Cell Biology Unit1B4.

Goossens E \& Tournaye H 2007 Is there a clinical future for spermatogonial stem cells? Current Stem Cell Research \& Therapy 2 189-195.

Gourdon JC \& Travis AJ 2011 Spermatogenesis in ferret testis xenografts: a new model. Comparative Medicine 61 145-149.

Herrid M, Vignarajan S, Davey R, Dobrinski I \& Hill JR 2006 Successful transplantation of bovine testicular cells to heterologous recipients. Reproduction 132 617-624.

Hochereau-de-Reviers MT \& Perreau C 1997 Induced differentiation of ovine foetal gonocytes after grafting in the scrotum of nude mice. Reproduction Nutrition Development 37 469-476.

Honaramooz A 2012 Cryopreservation of Testicular Tissue. In Current Frontiers in Cryobiology, pp 209-228. Ed I Katkov. Rijeka: InTech Publication.

Honaramooz A \& Yang Y 2011 Recent advances in application of male germ cell transplantation in farm animals. Veterinary Medicine International 657860 1-9.

Honaramooz A, Snedaker A, Boiani M, Schöler H, Dobrinski I \& Schlatt S 2002a Sperm from neonatal mammalian testes grafted in mice. Nature 418 778-781.

Honaramooz A, Megee SO \& Dobrinski I 2002b Germ cell transplantation in pigs. Biology of Reproduction 66 21-28.

Honaramooz A, Behboodi E, Blash S, Megee SO \& Dobrinski I 2003a Germ cell transplantation in goats. Molecular Reproduction and Development 64 422-428.

Honaramooz A, Behboodi E, Megee SO, Overton SA, Galantino-Homer H, Echelard Y \& Dobrinski I 2003b Fertility and germline transmission of donor haplotype following germ cell transplantation in immunocompetent goats. Biology of Reproduction 69 1260-1264.
Honaramooz A, Li MW, Penedo MCT, Meyers S \& Dobrinski I 2004 Accelerated maturation of primate testis by xenografting into mice. Biology of Reproduction 70 1500-1503.

Honaramooz A, Zeng W, Rathi R, Koster J, Ryder O \& Dobrinski I 2005 Testis tissue xenografting to preserve germ cells from a cloned banteng calf. Reproduction, Fertility, and Development 17247.

Honaramooz A, Megee SO, Rathi R \& Dobrinski I 2007 Building a testis: formation of functional testis tissue after transplantation of isolated porcine (Sus scrofa) testis cells. Biology of Reproduction 76 43-47.

Honaramooz A, Cui XS, Kim NH \& Dobrinski I 2008 Porcine embryos produced after intracytoplasmic sperm injection using xenogeneic pig sperm from neonatal testis tissue grafted in mice. Reproduction, Fertility, and Development 20 802-807.

Hou M, Andersson M, Eksborg S, Soder O \& Jahnukainen K 2007 Xenotransplantation of testicular tissue into nude mice can be used for detecting leukemic cell contamination. Human Reproduction 22 1899-1906.

Huang S, Sartini BL \& Parks JE 2008 Spermatogenesis in testis xenografts grafted from pre-pubertal Holstein bulls is re-established by stem cell or early spermatogonia. Animal Reproductive Science 103 1-12.

IUCN 2013 IUCN Red List of Threatened Species. Version 2013.2. Available from: http://www.iucnredlist.org. Downloaded on 8 April 2014.

Izadyar F, Den Ouden K, Stout TA, Stout J, Coret J, Lankveld DP, Spoormakers TJ, Colenbrander B, Oldenbroek JK, Van der Ploeg KD, Woelders H, Kal HB \& de Rooij DG 2003 Autologous and homologous transplantation of bovine spermatogonial stem cells. Reproduction 126 765-774.

Jahnukainen K, Ehmcke J, Hergenrother SD \& Schlatt S 2007a Effect of cold storage and cryopreservation of immature non-human primate testicular tissue on spermatogonial stem cell potential in xenografts. Human Reproduction 22 1060-1067.

Jahnukainen K, Ehmcke J, Nurmio M \& Schlatt S 2007b Irradiation causes acute and long-term spermatogonial depletion in cultured and xenotransplanted testicular tissue from juvenile nonhuman primates. Endocrinology 148 5541-5548.

Johnson L, Suggs LC, Norton YM \& Zeh WC 1996 Effect of developmental age or time after transplantation on Sertoli cell number and testicular size in inbred Fischer rats. Biology of Reproduction 54 948-959.

Keros V, Hultenby K, Borgström B, Fridström M, Jahnukainen K \& Hovatta O 2007 Methods of cryopreservation of testicular tissue with viable spermatogonia in pre-pubertal boys undergoing gonadotoxic cancer treatment. Human Reproduction 22 1384-1395.

Kim Y, Selvaraj V, Pukazhenthi B \& Travis AJ 2007 Effect of donor age on success of spermatogenesis in feline testis xenografts. Reproduction, Fertility, and Development 19 869-876.

Kuopio T, Savouras PO, Pelliniemi LJ \& Huhtaniemi IT 1989 Transplantation of newborn rat testis under the kidney capsule of adult host as a model to study the structure 
and function of Leydig cells. Journal of Andrology $\mathbf{1 0}$ 335-345.

Mastromonaco GF \& King WA 2007 Cloning in companion animal, non-domestic and endangered species: can the technology become a practical reality? Reproduction, Fertility, and Development 19 748-761.

Meachem SJ, Stanton PG \& Schlatt S 2005 Folliclestimulating hormone regulates both Sertoli cell and spermatogonial populations in the adult photoinhibited djungarian hamster testis. Biology of Reproduction 72 1187-1193.

Mitchell RT, Saunders PTK, Childs AJ, Cassidy-Kojima C, Anderson RA, Wallace WHB, Kelnar CJH \& Sharpe RM 2010 Xenografting of human fetal testis tissue: a new approach to study fetal testis development and germ cell differentiation. Human Reproduction 25 2405-2414.

Mota PC, Ramalho-Santos J \& Schlatt S 2011 Xenografting as a tool to preserve endangered species: outcomes and challenges in model systems. Veterinary Medicine International 629409 1-7.

Nagano M, McCarrey JR \& Brinster RL 2001 Primate spermatogonial stem cells colonize mouse testes. Biology of Reproduction 64 1409-1416.

Nagano M, Patrizio P \& Brinster RL 2002 Long-term survival of human spermatogonial stem cells in mouse testes. Fertility and Sterility 78 1225-1233.

Nakai M, Kaneko H, Somfai T, Maedomari N, Ozawa M, Noguchi J, Ito J, Kashiwazaki N \& Kikuchi K 2010 Production of viable piglets for the first time using sperm derived from ectopic testicular xenografts. Reproduction 139 331-335.

Naughton CK, Jain S, Strickland AM, Gupta A \& Milbrandt J 2006 Glial cell-line derived neurotrophic factor-mediated RET signaling regulates spermatogonial stem cell fate. Biology of Reproduction 74 314-321.

Oatley JM, de Avila DM, Reeves JJ \& McLean DJ 2004 Spermatogenesis and germ cell transgene expression in xenografted bovine testicular tissue. Biology of Reproduction 71 494-501.

Oatley JM, Reeves JJ \& McLean DJ 2005 Establishment of spermatogenesis in neonatal bovine testicular tissue following ectopic xenografting varies with donor age. Biology of Reproduction 72 358-364.

Ogawa T, Dobrinski I, Avarbock MR \& Brinster RL 1999 Xenogeneic spermatogenesis following transplantation of hamster germ cells to mouse testes. Biology of Reproduction 60 515-521.

Ohta H \& Wakayama T 2005 Generation of normal progeny by intracytoplasmic sperm injection following grafting of testicular tissue from cloned mice that dies postnatally. Biology of Reproduction 73 390-395.

Orwig KE \& Schlatt S 2005 Cryopreservation and transplantation of spermatogonia and testicular tissue for preservation of male fertility. Journal of National Cancer Institute Monogram 2005 51-56.

Paris MCJ \& Schlatt S 2007 Ovarian and testicular tissue xenografting: its potential for germline preservation of companion animals, non-domestic and endangered species. Reproduction, Fertility, and Development 19 771-782.
Rathi R, Honaramooz A, Zeng W, Schlatt S \& Dobrinski I 2005 Germ cell fate and seminiferous tubule development in bovine testis xenografts. Reproduction 130 923-929.

Rathi R, Honaramooz A, Zeng W, Turner R \& Dobrinski I 2006 Germ cell development in equine testis tissue xenografted into mice. Reproduction 131 1091-1098.

Rathi R, Zeng W, Megee S, Conley A, Meyers S \& Dobrinski I 2008 Maturation of testicular tissue from infant monkeys after xenografting into mice. Endocrinology 149 5288-5296.

Reddy N, Mahla RS, Thathi R, Suman SK, Jose J \& Goel S 2012 Gonadal status of male recipient mice influences germ cell development in immature buffalo testis tissue xeonograft. Reproduction 143 59-69.

Rodriguez-Sosa JR \& Dobrinski I 2009 Recent developments in testis tissue xenografting. Reproduction 138 187-194.

Rodriguez-Sosa JR, Dobson H \& Dobrinski I 2006 Isolation and transplantation of spermatogonia in sheep. Theriogenology 66 2091-2103.

Rodriguez-Sosa JR, Foster RA \& Hahnel A 2010 Development of strips of ovine testes after xenografting under the skin of mice and co-transplantation of exogenous spermatogonia with grafts. Reproduction 139 227-235.

Rodriguez-Sosa JR, Rathi R, Wang Z \& Dobrinski I 2011 Development of bovine fetal testis tissue after ectopic xenografting in mice. Journal of Andrology 32 271-281.

Rodriguez-Sosa JR, Costa GM, Rathi R, Franca LR \& Dobrinski I 2012 Endocrine modulation of the recipient environment affects development of bovine testis tissue ectopically grafted in mice. Reproduction 144 37-51.

Sato T, Katagiri K, Gohbara A, Inoue K, Ogonuki N, Ogura A, Kubota Y \& Ogawa T 2011 In vitro production of functional sperm in cultured neonatal mouse testes. Nature 471 504-507.

Sato Y, Nozawa S, Yoshiike M, Arai M, Sasaki C \& Iwamoto T 2010 Xenografting of testicular tissue from an infant human donor results in accelerated testicular maturation. Human Reproduction 25 1113-1122.

Schlatt S, Kim SS \& Gosden R 2002 Spermatogenesis and steroidogenesis in mouse, hamster and monkey testicular tissue after cryopreservation and heterotopic grafting to castrated hosts. Reproduction 124 339-346.

Schlatt S, Honaramooz A, Boinai M, Schöler HR \& Dobrinski I 2003 Progeny from sperm obtained after ectopic grafting of neonatal mouse testes. Biology of Reproduction $\mathbf{6 8}$ 2331-2335.

Schlatt S, Honaramooz A, Ehmcke J, Goebell PJ, Rübben H, Dhir R, Dobrinski I \& Patrizio P 2006 Limited survival of adult human testicular tissue as ectopic xenograft. Human Reproduction 21 384-389.

Schlatt S, Westernströer B, Gassei K \& Ehmcke J 2010 Donorhost involvement in immature rat testis xenografting into nude mouse hosts. Biology of Reproduction 82 888-895.

Schmidt JA, de Avila JM \& McLean DJ 2006a Grafting period and donor age affect the potential for spermatogenesis in bovine ectopic testis xenografts. Biology of Reproduction 75 160-166.

Schmidt JA, de Avila JM \& McLean DJ 2006b Effect of vascular endothelial growth factor and testis tissue culture on spermatogenesis in bovine ectopic testis tissue xenografts. Biology of Reproduction 75 167-175. 
Schmidt JA, de Avila JM \& McLean DJ 2007 Analysis of gene expression in bovine testis tissue prior to ectopic testis tissue xenografting and during the grafting period. Biology of Reproduction 76 1071-1080.

Setchell BP 1990 The testis and tissue transplantation: historical aspects. Journal of Reproductive Immunology 18 1-8.

Shinohara T, Inoue K, Ogonuki N, Kanatsu-Shinohara M, Miki H, Nakata K, Kurome M, Nagashima H, Toyokuni S, Kogishi K, Honjo T \& Ogura A 2002 Birth of offspring following transplantation of cryopreserved immature pieces and in-vitro microinsemination. Human Reproduction 17 3039-3045.

Skakkebæk NE, Jensen G, Povlsen CO \& Rygaard J 1974 Heterotransplantation of human foetal testicular and ovarian tissue to the mouse mutant nude. Acta Obstetricia et Gynecologica Scandinavica Supplement 53 73-75.

Snedaker AK, Honaramooz A \& Dobrinski I 2004 A game of cat and mouse: xenografting of testis tissue from domestic kittens results in complete cat spermatogenesis in a mouse host. Journal of Andrology 25 926-930.

Tang L, Rodriguez-Sosa RJ \& Dobrinski I 2012 Germ cell transplantation and testis tissue xenografting in mice. Journal of Visualized Experiments 603545.

Van Saen D, Goossens E, Haentjens P, Baert Y \& Tournaye H 2013 Exogenous administration of recombinant human FSH does not improve germ cell survival in human prepubertal xenografts. Reproductive Biomedicine Online 26 286-298.

Vloeberghs V, Verheyen G \& Tournaye H 2013 Intracytoplasmic spermatid injection and in vitro maturation: fact or fiction? Clinics (Sao Paulo) 68 151-156.

Wyns C, Curaba M, Martinez-Madrid B, Van Langendonckt A, Francois-Xavier W \& Donnez J 2007 Spermatogonial survival after cryopreservation and short-term orthotopic immature human cryptorchid testicular tissue grafting to immunodeficient mice. Human Reproduction $\mathbf{2 2}$ 1603-1611.

Wyns C, Van Langendonckt A, Wese F-X, Donnez J \& Curaba M 2008 Long-term spermatogonial survival in cryopreserved and xenografted immature human testicular tissue. Human Reproduction 23 2402-2414.

Yang Y, Steeg J \& Honaramooz A 2010 The effects of tissue sample size and media on short-term hypothermic preservation of porcine testis tissue. Cell and Tissue Research 340 397-406.

Yu J, Cai Z-M, Wan H-J, Zhang F-T, Ye J, Fang J-Z, Gui Y-T \& Ye J-X 2006 Development of neonatal mouse and fetal human testicular tissue as ectopic grafts in immunodeficient mice. Asian Journal of Andrology $\mathbf{8}$ 393-403.

Zeng W, Avelar GF, Rathi R, Franca LR \& Dobrinski I 2006 The length of the spermatogenic cycle is conserved in porcine and ovine testis xenografts. Journal of Andrology 27 527-533.

Zeng W, Snedaker AK, Megee S, Rathi R, Chen F, Honaramooz A \& Dobrinski I 2009 Preservation and transplantation of porcine testis tissue. Reproduction, Fertility, and Development 21 489-497.

Zeng W, Baumann C, Schmidtmann A, Honaramooz A, Tang L, Bondareva A, Dores C, Fan T, Xi S, Geiman T, Rathi R, de Rooij D, De La Fuente R, Muegge K \& Dobrinski I 2011 Lymphoid-specific helicase (HELLS) is essential for meiotic progression in mouse spermatocytes. Biology of Reproduction 84 1235-1241. 
